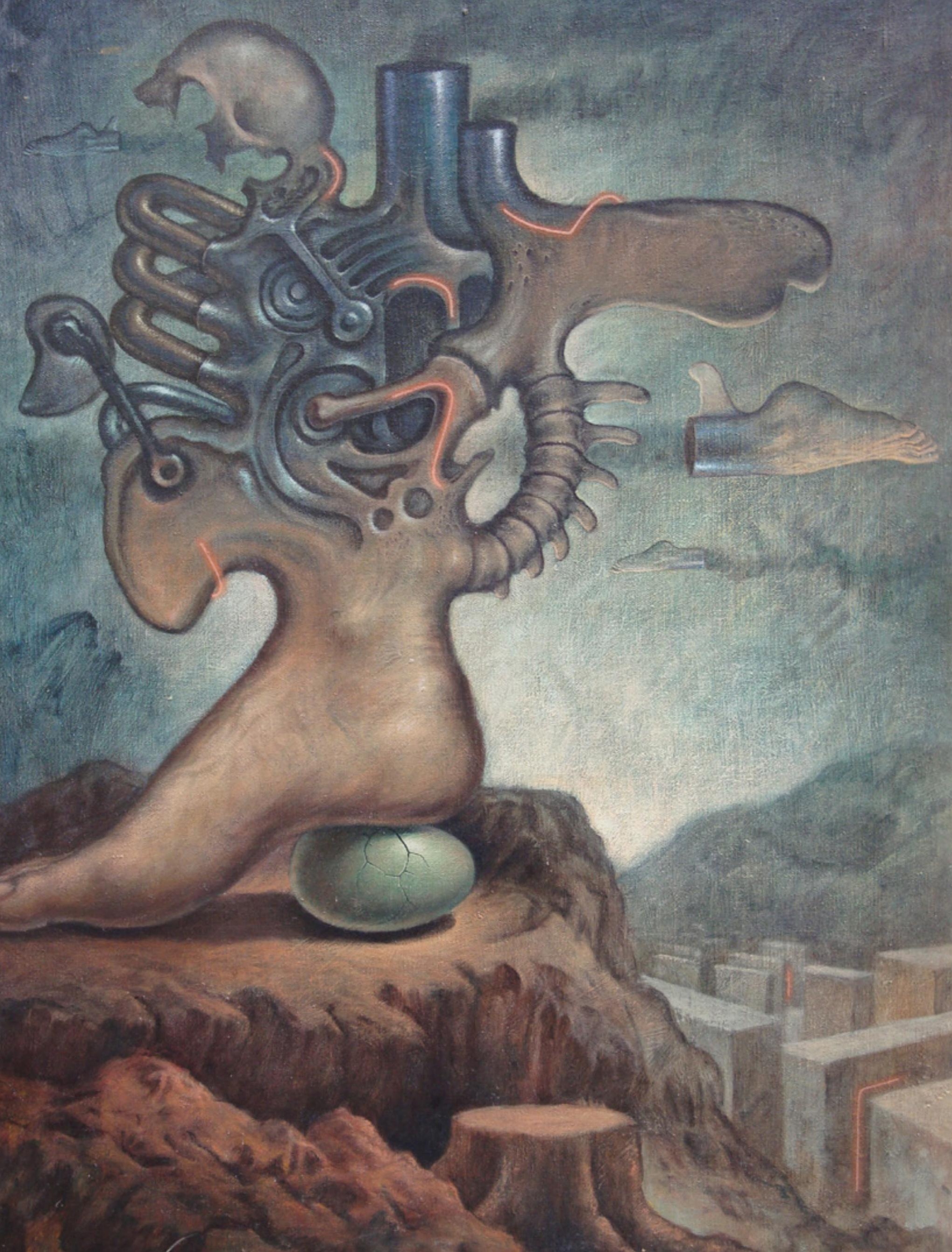




\section{La reprobación escolar en el nivel medio en el municipio de La Ceiba 2005-2007 \\ Ana Cecilia Ávila}

\section{INTRODUCCIÓN}

Esta investigación es un estudio de carácter descriptivo y se hace en el marco del Diplomado en Objetivos de Desarrollo del Milenio con Énfasis en Análisis Sociodemográfico enfocado en el tema "La reprobación de estudiantes del nivel medio según área de residencia periodo 2005-2007"

El trabajo consta de 6 capítulos: introducción, justificación, objetivos, metodología, historia del municipio de La Ceiba y reprobación de estudiantes del nivel medio en el municipio de La Ceiba según área de residencia período 2,005-2,007.

Este estudio tiene como propósito dar a conocer los datos de alumnos y alumnas que resultaron reprobados o aplazados en relación a la cantidad de alumnos matriculados al iniciar cada año académico del período 2,005-2,007.

Esta investigación provee de información importante para el diagnóstico de la realidad educativa y pretende facilitar el diseño, seguimiento y evaluación de planes y programas de apoyo social y económico, orientados a mejorar el nivel educativo de la población.

Agradezco a todo el personal de la Maestría en Demografía Social los cuales lograron despertar el interés en hacer posible dicho estudio como ser las Licenciadas Marta Lorena Suazo, Marysabel Zelaya Ochoa, Licenciados Elvis Trejo Teruel, Jorge Plata Zelaya, Héctor Baca Santos, Dr. Raúl Samayoa Pineda, y todo el apoyo brindado por el Sr. Samuel Flores. Así mismo agradezco la colaboración recibida por parte de la Distrital de Educación de la ciudad de La Ceiba por medio del Sr. Bernardo Oviedo Banegas de la Unidad de Planificación y Evaluación de
Gestión; a mi jefe del Departamento de Investigación y Estadística de la municipalidad de La Ceiba Ing. José Rafael Sosa, quien me brindó todo su apoyo para poder asistir a este Diplomado en Objetivos de Desarrollo del Milenio con Énfasis en Análisis Sociodemográfico.

\section{II.- JUSTIFICACIÓN}

Uno de los grandes problemas que tiene la educación en Honduras es el alto índice de reprobación que se da en todos los niveles educativos.

En el caso de la educación media, la reprobación sigue siendo uno de los grandes frenos que impiden la culminación satisfactoria de los alumnos y alumnas de este nivel. Los factores directamente relacionados con la reprobación van desde una base escolar deficiente, carencias económicas que obligan al estudiante a ocupar gran parte de su tiempo en algún tipo de trabajo y falta de acceso a la información, entre otras.

Es de suma importancia conocer los resultados anuales de reprobación ya que es un problema que tiene repercusión en el nivel medio que es cuando el alumno o alumna toma la decisión de acuerdo a sus aptitudes y capacidades para orientarse hacia la profesión que le interese ejercer en un futuro, también por el gasto que representa para el Estado la reprobación del año lectivo, al igual que el gasto que represeta para sus padres por la inversión hecha durante el año y seguidamente en el pago de las clases extras que reciben los reprobados en la Escuela Vacacional (Llamada popularmente "La Escuelita").

Bajo ese contexto la realización de un estudio que muestre los porcentajes de reprobación en el nivel medio según área de residencia es de suma importancia porque 
permitirá identificar las causas y factores asociados a este fenómeno.

\section{III.- OBJETIVOS}

\section{Objetivo general:}

- Analizar datos de reprobados del nivel medio en el municipio de La Ceiba según el área de residencia en los años 2,005 - 2,007.

\section{Objetivos específicos:}

- Describir el número de instituciones educativas, de acuerdo al número de alumnos y alumnas matriculadas y número de docentes por año en el nivel de educación media.

- Comparar los datos de matrícula y reprobación, que se registran en el área rural y urbana en el nivel medio en el municipio de La Ceiba en los años 2,005-2,007.

\section{IV.- METODOLOGÍA}

Para la realización del estudio sobre "La reprobación de estudiantes en el nivel medio según área de residencia en el municipio de La Ceiba", el método utilizado consistió en una investigación documental

Para obtener la información se pasó por varias fases:

- Recopilación de información secundaria en el Dirección Distrital de Educación de Las Ceiba, departamento de Investigación y Estadística Municipal DIEM

- Entrevistas a alumnos, alumnas y maestros de algunos centros educativos.

- Lectura de datos estadísticos y elaboración de tablas y gráficos.

\section{V.- MUNICIPIO DELA CEIBA}

\subsection{Ubicación geográfica del municipio de La Ceiba}

El municipio de La Ceiba tiene una extensión territorial de $639.45 \mathrm{Km}^{2}$, de los cuales $73.33 \mathrm{Km}^{2}$ son considerados área urbana municipal y $566.23 \mathrm{Km}^{2}$ es área rural (Instituto Geográfico Nacional de Honduras, 1998)
Los límites físicos del municipio de La Ceiba son los siguientes:

Al norte: con el Mar Caribe o de Las Antillas.

Al sur: con municipio de Olanchito (Depto. de Yoro).

Al este: con municipio de Jutiapa (Depto. de Atlántida).

Al oeste: con municipio de El Porvenir (Depto. de Atlántida).

Según los límites matemáticos el municipio de La Ceiba está ubicada a: $15^{\circ} 32^{\prime} 34^{\prime \prime}$ de latitud norte y $86^{\circ}$ 53' 17" de longitud oeste.

La ciudad de La Ceiba se encuentra a una altura de18 msnm.

La ciudad de La Ceiba geográficamente está dividida en tres sectores:

El Sector 1 (Oeste), está ubicado entre el Río Danto y el Río Bonito, tiene una extensión superficial de 2,053 hectáreas. Este sector posee una estructura de desarrollo lineal cuyo eje principal es la carretera que conduce de La Ceiba hacia Tela, implementando una serie de actividades industriales y comerciales. Los principales polos de apoyo al desarrollo se deben a las actividades generalizadas por el Aeropuerto Golosón y el Centro Universitario Regional del Litoral Atlántico (CURLA), cuya superficie es de alrededor 400 hectáreas.

El Sector 2 (Centro), está ubicado entre el Río Cangrejal y el Río Danto, tiene una extensión superficial de 1,491 hectáreas. Este sector está formado por el antiguo casco urbano de la ciudad y es donde se concentran las actividades económicas e institucionales de la ciudad.

El Sector 3 (Este), está ubicado entre el Río Juana Leandra o Los Chorros y el Río Cangrejal, tiene una extensión superficial de 3,778 hectáreas. Debido a la ampliación del perímetro urbano y el acceso al Departamento de Colón, está presentando una mayor característica de desarrollo habitacional.

1.2 Crecimiento y proyección de la población rural y urbana del municipio de La Ceiba 1,974; 1,988 y 2001 al 2010

Según proyecciones realizadas por el INE entre 1974 y 
Gráfico No. 1

Municipio de La Ceiba, según sectores

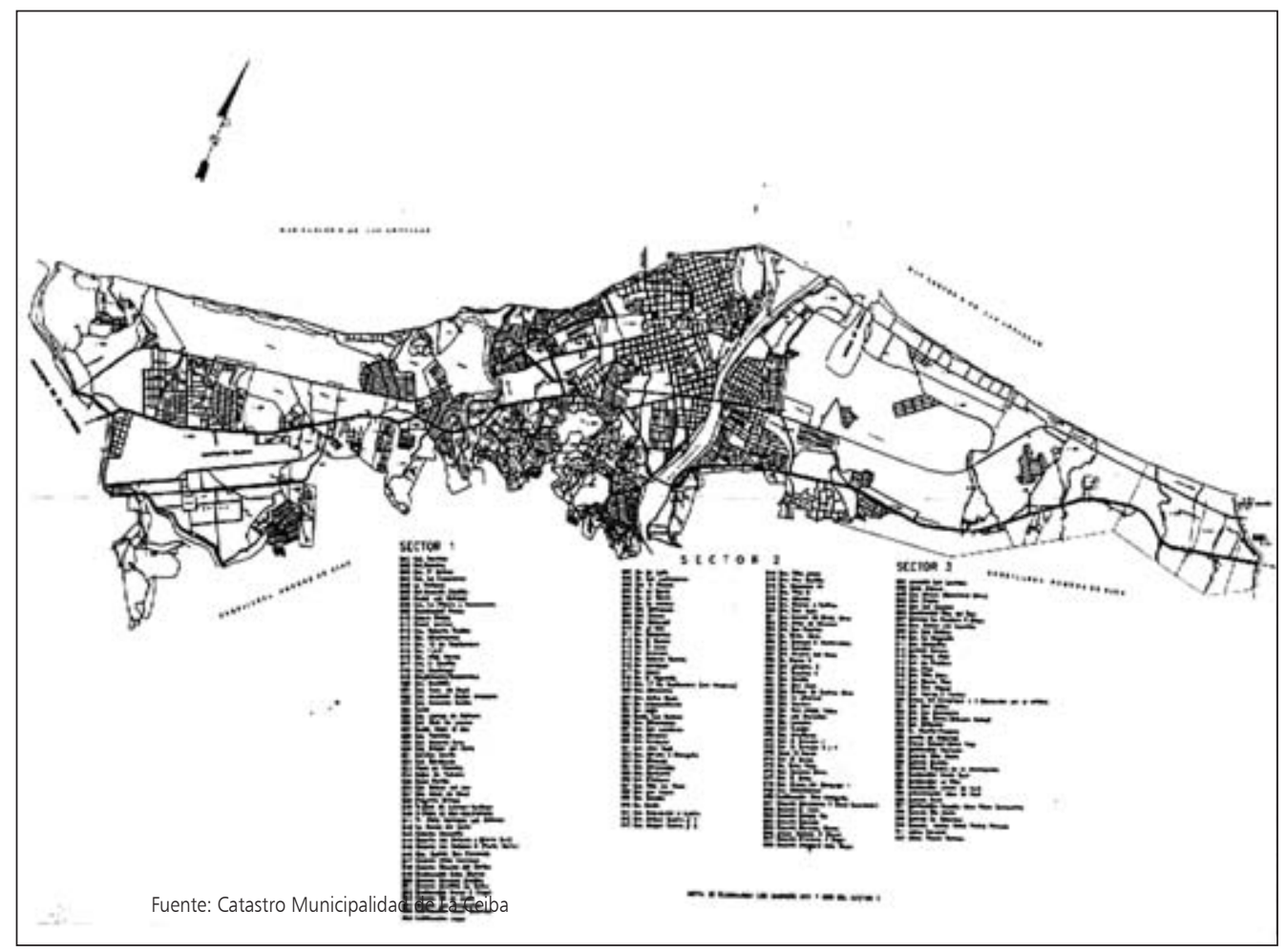

Catastro Municipalidad de La Ceiba, 2001.

el 2010 el área urbana del municipio de La Ceiba concentra el $93.1 \%$ de la población total, y el área rural 6.9\%. Es importante hacer notar que el área urbana tiene una extensión territorial de $73.22 \mathrm{Km}^{2}$, aproximadamente siete veces menor que el área rural con $566.23 \mathrm{Km}^{2}$. La población del municipio a pesar de la importante concentración urbana en La Ceiba, es mayoritariamente rural. (Cuadro No. 1)

\section{REPROBACION DE ESTUDIANTES DEL NIVEL ME- DIO EN EL MUNICIPIO DE LA CEIBA, SEGÚN ÁREA DE RESIDENCIA PERIODO 2,005-2,007}

\subsection{La Educación en el municipio de La Ceiba}

En la ciudad de La Ceiba la municipalidad ha realizado una significativa inversión en educación, no obstante el monto de la inversión y las acciones emprendidas para mejorar los índices, no han sido suficientes para contrarrestar la vulnerabilidad que todavía presenta el sector
Cuadro No. 1. Municipio de La Ceiba: proyección poblacional según área de residencia 1974-2010

\begin{tabular}{|c|c|c|c|}
\hline AÑO2 & TOTAL & $\begin{array}{c}\text { POBLACIÓN } \\
\text { RURAL } \\
\mathbf{( 6 . 9 \% )}\end{array}$ & $\begin{array}{c}\text { POBLACIÓN } \\
\text { URBANA } \\
\mathbf{( 9 3 . 1 \% )}\end{array}$ \\
\hline $\mathrm{a} 1,974$ & 47,835 & 9,047 & 38,788 \\
\hline $\mathrm{b} 1,988$ & 80,603 & 9,935 & 70,668 \\
\hline 2,001 & 139,229 & 9,653 & 129,576 \\
\hline 2,002 & 144,187 & 9,403 & 134,784 \\
\hline 2,003 & 149,216 & 9,153 & 140,063 \\
\hline 2,004 & 154,31 & 8,903 & 145,407 \\
\hline 2,005 & 159,465 & 8,654 & 150,811 \\
\hline 2,006 & 164,671 & 8,405 & 156,266 \\
\hline 2,007 & 169,922 & 8,156 & 161,766 \\
\hline 2,008 & 175,206 & 7,908 & 167,298 \\
\hline 2,009 & 180,512 & 7,662 & 172,85 \\
\hline 2,01 & 185,831 & 7,416 & 178,415 \\
\hline
\end{tabular}

Fuente: Censo Nacional de1974 Censo Nacional de 1988, Estimación de la Población por municipio, Área Rural y Urbana establecida por el INE según Censo Nacional del 2001.

educativo, en el mejoramiento de la calidad de servicios que se ofrecen. 
Se debe garantizar la educación con calidad, lo cual es responsabilidad de la Secretaría de Educación. Cumplir este derecho a toda la población requiere para unos países, más que para otros, un gran esfuerzo.

El municipio de La Ceiba tiene una cobertura escolar en los cuatro niveles de la educación: Pre-escolar, Primaria, Básico (1ํ-9ํ), Medio y Superior.

La mayor cobertura del sector público se encuentra en el sector de Educación Primaria, similar a la tendencia nacional. En relación a la matrícula de alumnos y alumnas estos se concentran en el área urbana tanto en el sector público como privado. De igual manera predominan los centros escolares del sector público y consecuentemente el mayor número de docentes.

El nivel medio funciona bajo la modalidad presencial y a distancia a través de los ISEMED y el Sistema de Aprendizaje Tutorial (SAT)

En la ciudad de La Ceiba actualmente funcionan 37 instituciones de Educación Media de los cuales:

* 4 son instituciones urbanas oficiales

* 26 son instituciones urbanas privadas

* 3 son instituciones rurales oficiales

* 1 es institución rural privada

* 1 es de educación media a distancia

Tabla No. 2. Municipio de La Ceiba: instituciones educativas por área de residencia en el 2008

\begin{tabular}{|c|c|c|c|c|}
\hline \multirow[t]{2}{*}{ Instituciones Educativas } & \multicolumn{2}{|c|}{ Públicas } & \multicolumn{2}{|c|}{ Privadas } \\
\hline & RURALES & URBANAS & RURALES & URBANAS \\
\hline \multicolumn{5}{|l|}{$\underline{\text { Jardines de Niños }}$} \\
\hline Número de Instituciones & 8 & 41 & 0 & 36 \\
\hline Número de Docentes & 5 & 48 & 0 & 92 \\
\hline Número de Estudiantes & 150 & 1,600 & 0 & 1,819 \\
\hline \multicolumn{5}{|l|}{ Escuelas Primarias } \\
\hline Número de Instituciones & 32 & 39 & 0 & 40 \\
\hline Número de Docentes & 100 & 436 & 0 & 343 \\
\hline Número de Estudiantes & 2,978 & 12,183 & 0 & 5,655 \\
\hline \multicolumn{5}{|l|}{$\frac{\text { Centros de Educación }}{\text { Básica General }}$} \\
\hline Número de Instituciones & 2 & 10 & 0 & 0 \\
\hline Número de Docentes & 22 & 244 & 0 & 0 \\
\hline Número de Estudiantes & 555 & 7,853 & 0 & 0 \\
\hline \multicolumn{5}{|l|}{$\underline{\text { Institutos Secundarios }}$} \\
\hline Número de Instituciones & 3 & 4 & 1 & 26 \\
\hline Número de Docentes & 43 & 257 & 11 & 497 \\
\hline Número de Estudiantes & 681 & 7,249 & 273 & 8,471 \\
\hline \multicolumn{5}{|l|}{$\underline{\text { Instituto a Distancia (Isemed) }}$} \\
\hline Número de Instituciones & 0 & 1 & 0 & 0 \\
\hline Número de Docentes & 0 & 24 & 0 & 0 \\
\hline Número de Estudiantes & 0 & 552 & 0 & 0 \\
\hline \multicolumn{5}{|l|}{ Escuelas Nocturnas } \\
\hline Número de Instituciones & 0 & 4 & 0 & 0 \\
\hline Número de Docentes & 0 & 18 & 0 & 0 \\
\hline Número de Estudiantes & 0 & 243 & 0 & 0 \\
\hline
\end{tabular}

Fuente: Dirección Distrital de Educación de La Ceiba, 2008. 
* 2 son instituciones alternativas de educación técnica

La educación debe de ser un elemento clave para el desarrollo en el municipio de La Ceiba, y debe tener como premisa, la formación del capital humano con las herramientas necesarias para enfrentar los retos que supone en la actualidad un mundo competitivo y globalizado.

Es destacable el hecho de que La Ceiba, es uno de los municipios que cuenta con mayor tasa de alfabetización en Honduras, lo que le otorga un fuerte potencial para alcanzar un buen nivel de desarrollo sostenible.

La educación es un aspecto clave de desarrollo, un elemento a través del cual las sociedades tienen la posibilidad de desarrollarse y de consolidarse, a través de la instrucción de las nuevas generaciones se busca la formación del capital humano que permita a la sociedad un desarrollo sostenible y equilibrado, especialmente en la época actual en la que vivimos un proceso de globalización y de competitividad.

\subsection{Matrícula en el municipio de La Ceiba}

La matrícula está referida al porcentaje de hombres y mujeres inscritos en los niveles de enseñanza primario, secundario y terciario en escuelas públicas y privadas.

La matrícula gratis medida implementada en el Gobierno Sr. José Manuel Zelaya ayudó a incrementar la matrícula de estudiantes en un $28 \%$ en cuatro años pasando de 1.8 millones en 2005 a 2.3 millones en 2009, esto permitió que medio millón de niños y niñas que no tenían acceso, pudieron ingresar al sistema educativo y ha beneficiado al 95\% de los centros educativos públicos a mediados del 2009 (Secretaría de Educación, 2009).

En relación a la matrícula escolar del municipio de La Ceiba en el nivel medio en los tres últimos años se observa de manera general una tendencia al crecimiento entre el $5 \%$ y $7 \%$, situación bastante positiva para la población en edad escolar puesto que abre la oportunidad de adquirir conocimientos básicos para su desempeño profesional.
Tabla No. 3. Municipio de La Ceiba: matrícula escolar del nivel medio 2005-2007

\begin{tabular}{|l|c|c|c|}
\hline \multicolumn{1}{|c|}{ Nivel medio } & $\begin{array}{c}\text { Matrícula } \\
\mathbf{2 0 0 5}\end{array}$ & $\begin{array}{c}\text { Matrícula } \\
\mathbf{2 0 0 6}\end{array}$ & $\begin{array}{c}\text { Matrícula } \\
\mathbf{2 0 0 7}\end{array}$ \\
\hline Ciclo común & 7,462 & 7,849 & 8,317 \\
\hline Ciclo diversificado & 7,175 & 7,930 & 8,194 \\
\hline Total General & 14,637 & 15,779 & 16,511 \\
\hline
\end{tabular}

Fuente: Dirección Distrital de Educación de La Ceiba.

\section{Gráfico No. 1 Municipio de La Ceiba: matrícula escolar del nivel medio 2005-2007}

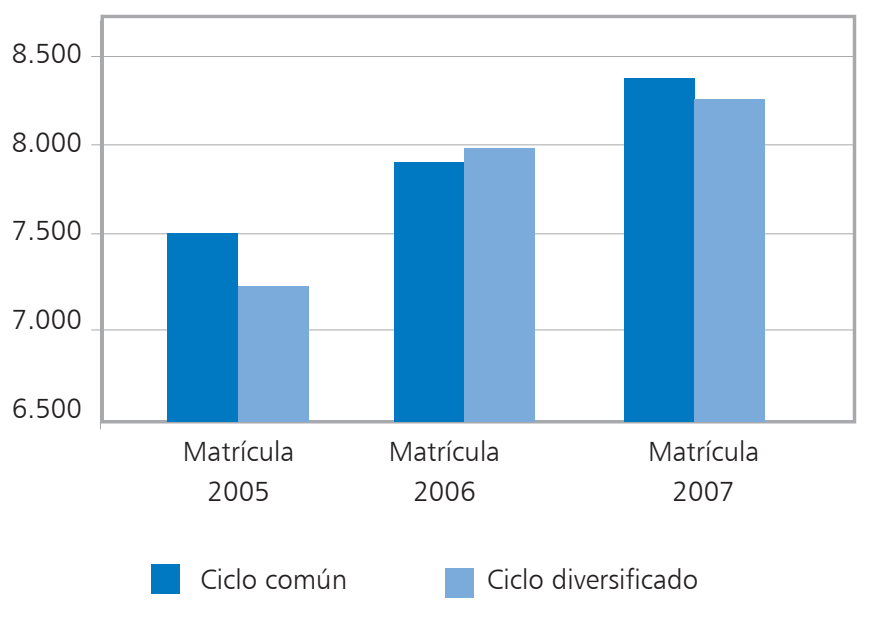

Fuente: Dirección Distrital de Educación de La Ceiba.

Un aspecto que vale la pena señalar es que aunque la matrícula escolar en el nivel medio ha aumentado el número de docentes no se ha incrementado en la misma proporción lo que provoca en alguna medida una sobrecarga en el número de alumnos por aula, cursos sin docentes asignados, factores que afectan la calidad de la educación en este nivel.

Tabla No. 4. Municipio de La Ceiba: docentes por nivel escolar 2005- 2007

\begin{tabular}{|l|c|c|c|}
\hline$N^{\circ}$ de profesores & 2005 & 2006 & 2007 \\
\hline Nivel medio & 796 & 804 & 806 \\
\hline
\end{tabular}

Fuente: Dirección Distrital de Educación de La Ceiba

\subsection{Reprobación en el municipio de La Ceiba}

La Secretaría de Educación de Honduras tiene establecido la escala de calificaciones que se aplican en el sis- 
tema educativo primario y medio del país, escala que rige tanto el sistema público como el sistema privado. En ese aspecto, se consideran reprobados en los ciclos parciales o curso aquellos estudiantes que en su desempeño escolar no alcancen una calificación igual o superior a $60 \%$.

6.3.1 Resultados totales de reprobados en el municipio de La Ceiba 2005-2007

Durante el 2005 el total general de alumnos y alumnas matrículados fue de 14,637, de los cuales el 90.9\% aprobó su curso lectivo y un $9.1 \%$ reprobó su curso. Al analizar los datos teniendo en cuenta el nivel de ciclo común y ciclo diversificado, en el primero existe un $10.25 \%$ de alumnos reprobados en diferentes materias; en el caso del ciclo diversificado existe un $7.90 \%$ de alumnos reprobados.

Durante el 2006 el total general de alumnos y alumnas matrículados fue de 15, 779, de los cuales el $82.86 \%$ aprobó su curso y un $17.41 \%$ reprobó. En el caso del ciclo común un $18.36 \%$ de alumnos reprobó y un $81.64 \%$ de alumnos aprobó satisfactoriamente. En diversificado un 15.93\% de alumnos reprobó y un $84.07 \%$ de alumnos aprobó el curso.

Durante el 2007, el total general de alumnos y alumnas matrículados fue de 16, 511, de los cuales el 80.92\% aprobó su curso lectivo y un $19.08 \%$ reprobó su curso. En el caso del ciclo común un $22.54 \%$ de alumnos reprobó y un $81.64 \%$ de alumnos aprobó el curso. En diversificado un $15.57 \%$ de alumnos reprobó y un $84.07 \%$ de alumnos aprobó el curso.

Se refleja que los mayores porcentajes de reprobación se registran entre los alumnos y alumnas de ciclo común, además esta reprobación ha ido en ascenso en el 2005 se registró en ciclo común un 10\% de reprobación, para el 2006 aumento 8 puntos porcentuales y para el 2007, 5 puntos más.

En caso de ciclo diversificado en el 2005 se registró $8 \%$ de reprobación, para el 2006 aumento 6 puntos porcentuales y en el 2007 se mantuvo el mismo valor.

Los datos reflejan que la tendencia a la reprobación en el nivel medio tiende a crecer sobre todo en el caso del ciclo común, lo que debe hacer reflexionar a las autoridades educativas y docentes de los institutos con el propósito de detectar aquellos factores que inciden en la reprobación.

\section{Gráfico No. 2. Resultados totales de reprobados en el municipio de La Ceiba 2005-2007}

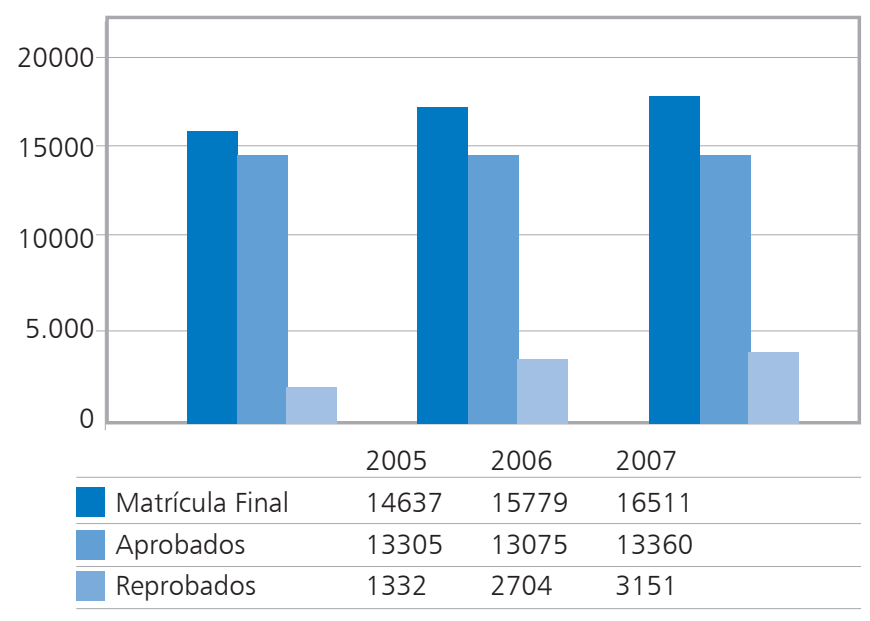

Fuente: Elaboración propia en base a datos de la Dirección Distrital de La Ceiba

6.3.2 Reprobados en institutos oficiales del área urbana, municipio de La Ceiba 2005-2007

Durante el 2005 el total general de alumnos y alumnas matrículados en institutos oficiales del área urbana fue de 5604 alumnos, de los cuales el 90.9\% aprobó su curso lectivo y un $9.1 \%$ reprobó su curso. $\mathrm{Al}$ analizar los datos teniendo en cuenta el nivel de ciclo común y ciclo diversificado, en el primero existe un $12.91 \%$ de alumnos reprobados en diferentes materias; en el caso del ciclo diversificado se presentó un $12.54 \%$ de alumnos reprobados.

Durante el 2006 el total general de alumnos y alumnas matrículados fue de 6430, de los cuales el $81 \%$ aprobó su curso lectivo y un 19\% reprobó su curso. En el caso del ciclo común existe un $18.36 \%$ de alumnos reprobados y un $81.64 \%$ de alumnos aprobados y en diversificado existe un $15.93 \%$ de alumnos reprobados y un $84.07 \%$ de alumnos aprobados.

Durante el 2007 el total general de alumnos y alumnas matrículados fue de 7239, de los cuales el 74\% aprobó su curso lectivo y un 26\% reprobó el curso. En el caso 
del ciclo común un $31.75 \%$ de alumnos reprobó y un 81.64\% de alumnos aprobó. En diversificado un 20.87\% de alumnos reprobó y un $84.07 \%$ de alumnos aprobó el curso. (Gráfico No. 3)

Gráfico No. 3. Reprobados en institutos oficiales del área urbana, municipio de La Ceiba 2005- 2007.

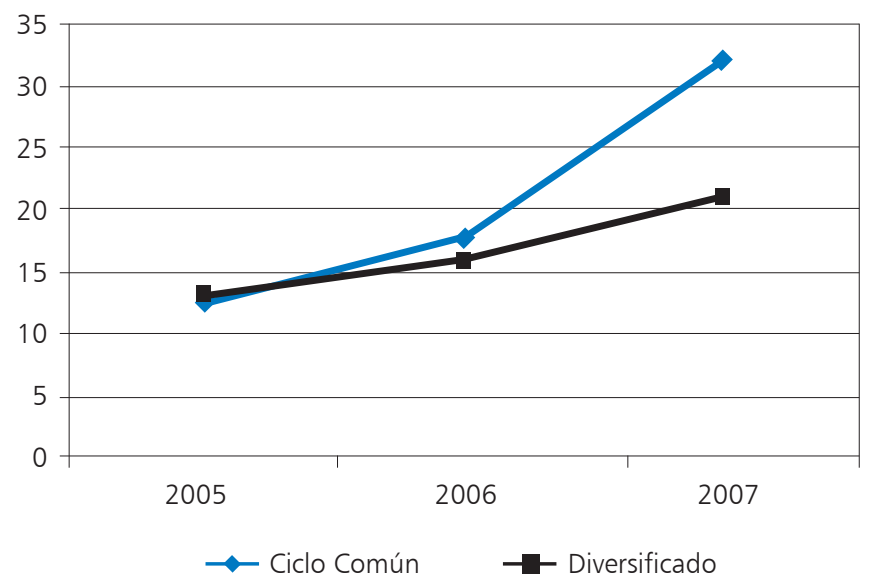

Fuente: Elaboración propia en base a datos de la Dirección Distrital de La Ceiba

6.3.3 Reprobados en institutos oficiales del área rural, municipio de La Ceiba

Del total general de 449 estudiantes de educación media de institutos oficiales del área rural se observa que en el transcurso del año lectivo 2,005 predominan 110 estudiantes que reprobaron diferentes materias representando un $24.50 \%$ del total, saliendo aprobados el restante $75.25 \%$, de los cuales, en ciclo común un 26.03\% de alumnos reprobó y un $73.97 \%$ de alumnos aprobó su curso. En diversificado un 17.86\% de alumnos reprobó y un $82.14 \%$ de alumnos aprobó el curso.

Del total general de 492 estudiantes de educación media de institutos oficiales del área rural se observa que en el transcurso del año lectivo 2,006 predominan 95 estudiantes que reprobaron diferentes materias representando un $19.31 \%$ del total, saliendo aprobados el restante $80.69 \%$, de los cuales, en ciclo común un $18.23 \%$ de alumnos reprobó y un $81.77 \%$ de alumnos aprobó. En diversificado un $23.15 \%$ de alumnos reprobó y un $76.85 \%$ de alumnos aprobó su curso.
Del total general de 746 estudiantes de educación media de institutos oficiales del área rural se observa que en el transcurso del año lectivo del 2,007 predominan 158 estudiantes que reprobaron diferentes materias siendo estos un $21.18 \%$ del total, saliendo aprobados el restante $78.82 \%$, de los cuales, en ciclo común un $22.14 \%$ de alumnos reprobó y un $77.86 \%$ de alumnos aprobó. En diversificado un $18.63 \%$ de alumnos reprobó y un $81.37 \%$ de alumnos aprobó el curso (Gráfico No. 4).

\section{Gráfico No. 4. Reprobados en institutos oficiales del área rural, municipio de La Ceiba}

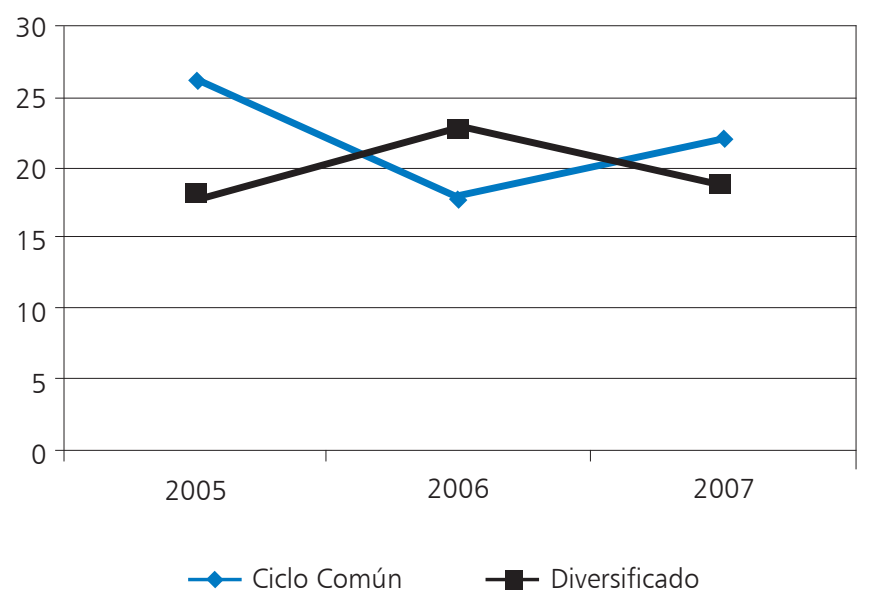

Fuente: Elaboración propia en base a datos de la Dirección Distrital de La Ceiba

6.3.4 Reprobados en institutos privados del área urbana, municipio de La Ceiba

Del total general de 7,792 estudiantes de educación media de institutos privados del área urbana se observa que en el transcurso del año lectivo 2,005 predominan 412 estudiantes que reprobaron diferentes materias representando un 5.29\% del total, saliendo aprobados el restante $94.71 \%$, de los cuales, en ciclo común un 5.88\% de alumnos reprobó y un $94.12 \%$ de alumnos aprobó.En diversificado un $4.72 \%$ de alumnos reprobó y un $95.28 \%$ de alumnos aprobó el curso.

Del total general de 7,797 estudiantes de educación media de institutos privados del área urbana se observa que en el transcurso del año lectivo 2,006 predominan 1,238 estudiantes que reprobaron diferentes materias 
representando un $15.88 \%$ del total, saliendo aprobados el restante $84.12 \%$, de los cuales, en ciclo común un $15.19 \%$ de alumnos reprobó y un $84.81 \%$ de alumnos aprobó. En diversificado existe un $16.49 \%$ de alumnos reprobó y un 83.51\% de alumnos aprobó el curso.

Del total general de 8,349 estudiantes de educación media de institutos privados del área urbana se observa que en el transcurso del año lectivo 2,007 predominan 1,091 estudiantes que reprobaron diferentes materias representando un $13.07 \%$ del total, saliendo aprobados el restante $86.93 \%$, de los cuales, en ciclo común un $15.33 \%$ de alumnos reprobó y un $84.67 \%$ de alumnos aprobó y en diversificado un $10.83 \%$ de alumnos reprobó y un $89.17 \%$ de alumnos aprobó el curso (Gráfico No. 5).

Gráfico No. 5. Municipio de La Ceiba: reprobados en educación media, institutos privados del área urbana 2005-2007

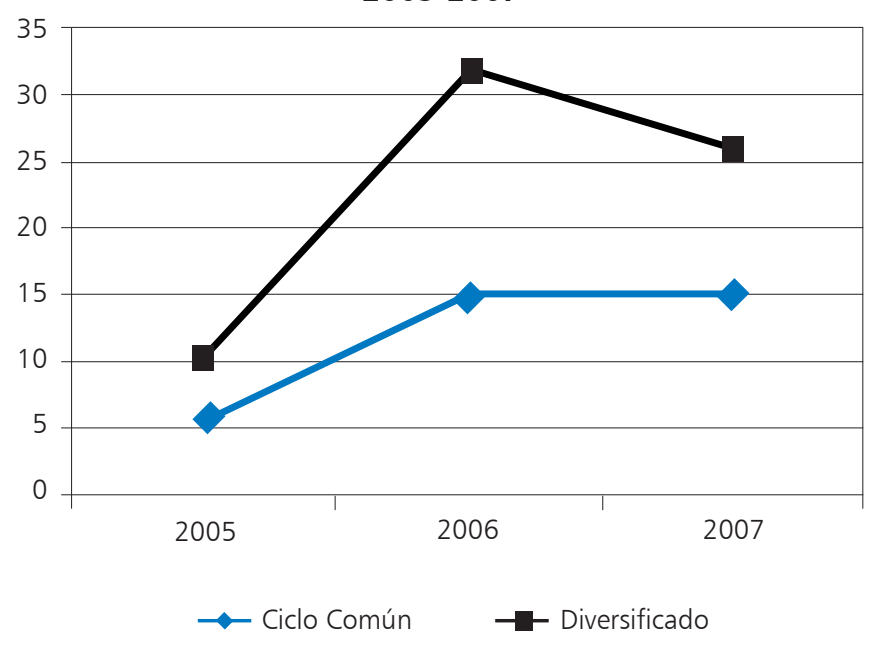

Fuente: Elaboración propia en base a los datos de la Dirección Distrital, 2008

6.3.5 Reprobados en institutos privados del área rural, municipio de La Ceiba

Del total general de137 estudiantes de educación media de institutos privados del área rural se observa que en el transcurso del año lectivo del 2,005 en su totalidad el $100 \%$ son estudiantes que aprobaron todas sus materias.

Del total general de178 estudiantes de educación media de institutos privados del área rural se observa que en el transcurso del año lectivo 2,006 en su tota- lidad el 100\% son estudiantes que aprobaron todas sus materias.

Del total general de177 estudiantes de educación media de institutos privados del área rural se observa que en el transcurso del año lectivo 2,007 prevalecen 10 estudiantes que reprobaron diferentes materias representando un $5.65 \%$ del total, saliendo aprobados el restante $94.35 \%$, de los cuales, en ciclo común un $4.96 \%$ de alumnos reprobó y un $95.04 \%$ de alumnos aprobó. En diversificado un $7.14 \%$ de alumnos reprobó y un $92.86 \%$ de alumnos aprobó el curso (Gráfico No. 6).

\section{Gráfico No. 6. Municipio de La Ceiba: reprobados en educación media, institutos privados del área rural}

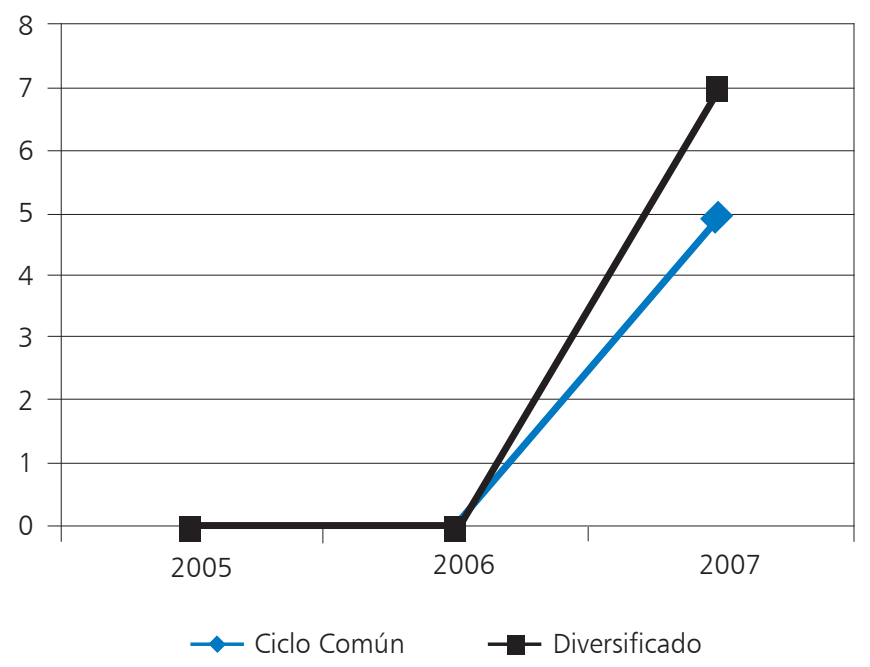

2005-2007

Fuente: Elaboración propia en base a los datos de la Dirección Distrital, 2008

6.3.6 Reprobados en ISEMED, municipio de La Ceiba Del total general de 573 estudiantes de educación media a distancia se observa que en el transcurso del año lectivo 2,005 predominan 97 estudiantes que reprobaron diferentes materias representando un 16.93\% del total, saliendo aprobados el restante $83.07 \%$, de los cuales, en ciclo común un $23.74 \%$ de alumnos reprobó y un $76.26 \%$ de alumnos aprobó. En diversificado un 5.58\% de alumnos reprobó y un $94.42 \%$ de alumnos que aprobó todas sus materias. 
Del total general de 685 estudiantes de educación media a distancia se observa que en el transcurso del año lectivo 2,006 predominan 100 estudiantes que reprobaron diferentes materias representado un $14.60 \%$ del total, saliendo aprobados el restante $85.40 \%$, de los cuales, en ciclo común un $13.60 \%$ de alumnos reprobó y un 86.40\% de alumnos aprobó. En diversificado un 15.97\% de alumnos reprobó y un $84.03 \%$ de alumnos que aprobó todas sus materias.

Del total general de 598 estudiantes de educación media a distancia se observa que en el transcurso del año lectivo 2,007 predominan 192 estudiantes que reprobaron diferentes materias representando un $32.11 \%$ del total, saliendo aprobados el restante $67.89 \%$, de los cuales, en ciclo común un $31.74 \%$ de alumnos reprobó y un $68.26 \%$ de alumnos aprobó. En diversificado un 32.58\% de alumnos reprobó y un $67.42 \%$ de alumnos que aprobó todas sus materias (Gráfico No. 7).

Gráfico No. 7. Municipio de La Ceiba: reprobados en educación media, ISEMED 2005-2007

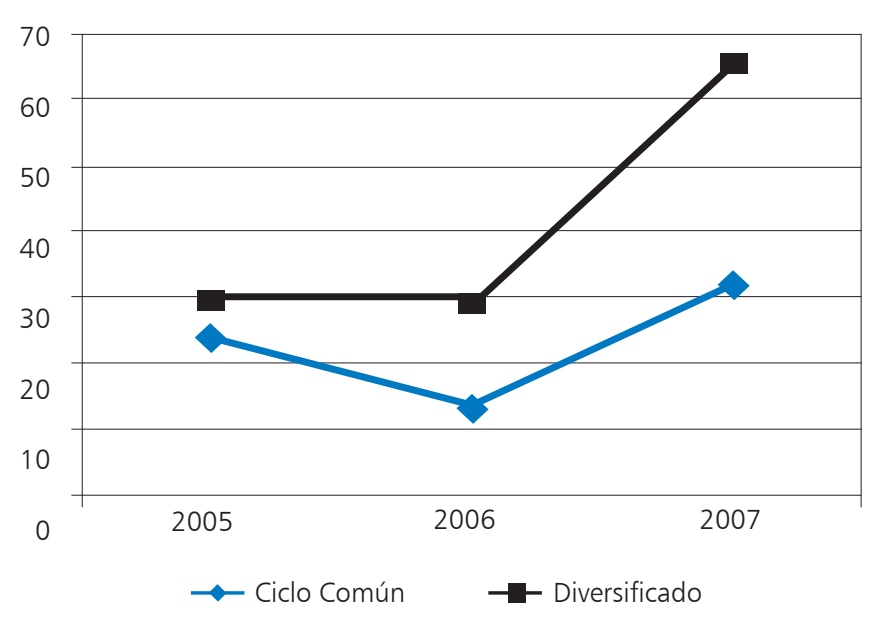

Fuente: Elaboración propia en base a los datos de la Dirección Distrital, 2008

6.3.7 Reprobados en Sistema de Aprendizaje Tutorial (SAT), municipio de La Ceiba,

Del total general de 82 estudiantes del Sistema de Aprendizaje Tutorial se observa que en el transcurso del año lectivo 2,005 predominan en su totalidad el 100\% que son estudiantes que aprobaron todas sus materias.
Del total general de114 estudiantes del Sistema de Aprendizaje Tutorial se observa que en el transcurso del año lectivo 2,006 predominan en su totalidad el 100\% de estudiantes que aprobaron todas sus materias.

Del total general de128 estudiantes del Sistema de Aprendizaje Tutorial se observa que en el transcurso del año lectivo 2,007 predominan 32 estudiantes que reprobaron diferentes materias siendo estos un $27.59 \%$ del total, saliendo aprobados el restante $72.41 \%$, de los cuales, en ciclo común un $27.59 \%$ de alumnos reprobó y un $72.41 \%$ de alumnos aprobó. En diversificado existe un $19.51 \%$ de alumnos reprobó y un $80.49 \%$ de alumnos que aprobó todas sus materias (Gráfico No. 8).

\section{Gráfico No. 8. Municipio de La Ceiba: reprobados en educación media, SAT 2005-2007}

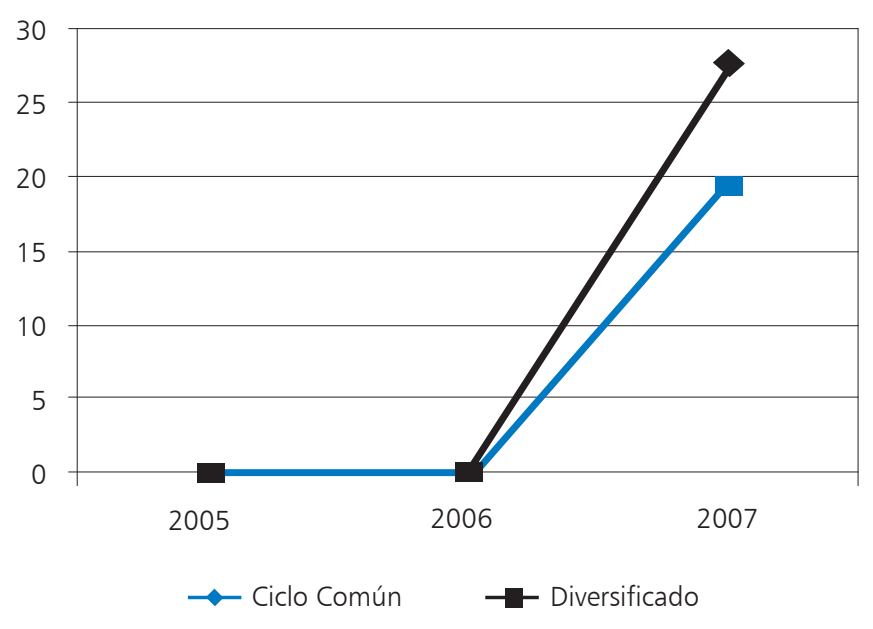

Fuente: Elaboración propia en base a los datos de la Dirección Distrital, 2008

\section{CONCLUSIONES}

- Uno de los aspectos positivos rescatados en el estudio es la tendencia al crecimiento de la matrícula en el nivel medio sobre todo en el sector público lo que está relacionado directamente con la implementación de la matrícula gratuita.

- De manera general se observa mayor reprobación entre alumnos y alumnas del sector público tanto en el área urbana como rural y específicamente en asignaturas 
como Matemáticas y Español, por lo que valdría la pena implementar acciones encaminadas a retroalimentar los conocimientos en estas asignaturas.

- Considerando el total de estudiantes matrículados anualmente en los años 2,005-2,007 se puede establecer que las causas que más inciden en la reprobación de las materias cursadas son: falta de apoyo y seguimiento por parte del departamento de orientación y consejería de tal manera que se puedan implementar a tiempo medidas que permitan a los estudiantes el aprovechamiento pleno de su año escolar, falta de apoyo de los padres en estar pendiente de cómo van académicamente sus hijos, fobia a los números y materias de bastante contenido teórico como Español y Estudios Sociales y falta de aplicación por parte de los y las estudiantes.

- Los indicadores demuestran que los proyectos y programas implementados en el municipio de La Ceiba no dan muy buen resultado en cuanto a la reprobación tomando en cuenta los datos de los años 2,005-2,007; donde se observa una menor reprobación en el año 2,005 con 1,332 jóvenes reprobados el cual ha ido en aumento ya que en el año 2,006 hubieron 2,704 reprobados y 3,151 jóvenes reprobados en el año 2,007.

VIII. BIBLIOGRAFÍA

- Listado de Centros Educativos y sus Características en el Nivel Medio

2,005-2,007 Dirección Distrital de Educación de La Ceiba. Lic. Gloria

Eloisa Medina - Sr. Bernardo Banegas

- Anuario Estadístico de la ciudad de La Ceiba, Atlántida, Departamento de Investigación y Estadística Municipal DIEM-LC 2,008.

- Mensajes de Ministro de Educación Marlon Brevé - Ley de Educación.

- Plan Estratégico de Desarrollo Municipal, Municipio de La Ceiba, Departamento Atlántida, Organización, Gemelos de Honduras.

- Secretaría de Educación. Dirección Distrital de Educación Municipio de La Ceiba. 2005-2007. 